\title{
Diffusion Imaging for Tumor Grading of Supratentorial Brain Tumors in the First Year of Life
}

\author{
S.F. Kralik, A. Taha, A.P. Kamer, J.S. Cardinal, T.A. Seltman, and C.Y. Ho
}

\begin{abstract}
BACKGROUND AND PURPOSE: Supratentorial tumors in the first year of life are typically large and heterogeneous at presentation, making differentiation of these CNS neoplasms on pre-operative imaging difficult. We hypothesize that the ADC value can reliably differentiate high- versus low-grade supratentorial tumors in this patient population.
\end{abstract}

MATERIALS AND METHODS: A blinded review of ADC maps was performed on 19 patients with histologically proved supratentorial brain tumors diagnosed within the first year of life. Minimum ADC values obtained by region of interest from 2 neuroradiologists were averaged and compared with World Health Organization tumor grade. ADC values for the entire tumor were also obtained by use of a semiautomated histogram method and compared with World Health Organization tumor grade. Data were analyzed by use of Spearman $\rho$ and Student $t$ test, with a value of $P<.05$ considered statistically significant.

RESULTS: For the manual ADC values, a significant negative correlation was found between the mean minimum $A D C$ and tumor grade $(P=$ .0016). A significant difference was found between the mean minimum ADC of the low-grade $\left(1.14 \times 10^{-3} \mathrm{~mm}^{2} / \mathrm{s} \pm 0.30\right)$ and high-grade tumors $\left(0.64 \times 10^{-3} \mathrm{~mm}^{2} / \mathrm{s} \pm 0.28\right)(P=.0018)$. Likewise, the semi-automated method demonstrated a significant negative correlation between the lowest 5th $(P=.0002)$ and 10th $(P=.0009)$ percentile individual tumor ADC values and tumor grade, a significant difference between the mean 5th and 10th percentile ADC values of the low-grade and high-grade groups $(P=.0028)$, and a significant positive correlation with values obtained by manual region-of-interest placement $(P<.000001)$.

CONCLUSIONS: ADC maps can differentiate high- versus low-grade neoplasms for supratentorial tumors presenting in the first year of life, given the significant negative correlation between ADC values and tumor grade.

ABBREVIATION: $W H O=$ World Health Organization

S upratentorial brain tumors in the first year of life are challenging from both a clinical and radiologic perspective. Clinical presentation is often delayed because of nonlocalizing symptoms and because the calvaria may accommodate increasing size of a mass and intracranial pressure at this age. ${ }^{1}$ Consequently, these tumors are often large on presentation, resulting in greater operative risks. A wide range of low-grade and high-grade pathologies may present within the first year of life. On radiologic examination, these tumors are typically large, heterogeneous, and may not demonstrate characteristic imaging features to

Received July 16, 2013; accepted after revision August 28

From Indiana University School of Medicine, Department of Radiology and Imaging Sciences, Indianapolis, Indiana.

Paper previously presented in part at: Annual Meeting of the Radiological Society of North America, November 24-30, 2012; Chicago, Illinois.

Please address correspondence to Chang Y. Ho, MD, 705 Riley Hospital Dr, MRI Department, Indianapolis, IN 46202; e-mail: cyho@iupui.edu

http://dx.doi.org/10.3174/ajnr.A3757 indicate a specific diagnosis. Imaging techniques that establish a more specific preoperative diagnosis would aid in surgical planning for gross or near total resection versus identification of locations for biopsy.

Diffusion imaging allows the evaluation of the diffusion of water in tissues. Quantitative analysis of the average diffusion rate in each voxel can be performed by use of the calculated ADC. There is a correlation between reduced diffusion of water and increasing tumor cellularity and therefore tumor grade. ${ }^{2}$ ADC values have been previously shown to reliably differentiate pediatric cerebellar tumors. ${ }^{3}$ We hypothesize that the ADC value can reliably differentiate high-grade versus low-grade supratentorial tumors in children presenting at an age of less than 1 year.

\section{MATERIALS AND METHODS}

After institutional review board approval was given, a retrospective radiology data base search from April 2003 to October 2011 
was performed on patients with age $\leq 1$ year who had brain MR imaging with a supratentorial mass confirmed by pathology. Postoperative pathologic diagnosis of the tumor type and World Health Organization (WHO) grade was recorded, and a blinded, retrospective review of the preoperative presentation brain MR imaging studies was performed. In cases that precede the most recent 2007 update for WHO tumor classification of the central nervous system, the pathologic report was analyzed for potential changes of WHO grading.

MR imaging was performed on 1.5T Signa LX (GE Healthcare, Milwaukee, Wisconsin), and $1.5 \mathrm{~T}$ and $3 \mathrm{~T}$ Avanto and Verio (Siemens, Erlangen, Germany) MR units with axial T2-weighted, fast spin-echo, sagittal and axial T1-weighted, axial fluid-attenuated inversion recovery, and postcontrast axial, coronal, and sagittal T1-weighted sequences. DWI was performed with multisection single-shot spin-echo echo-planar imaging with 5 -mm section thickness before administration of contrast material, with b-values of 0 and $1000 \mathrm{~s} / \mathrm{mm}^{2}$ applied in the $\mathrm{x}, \mathrm{y}$, and $\mathrm{z}$ directions. Processing of ADC maps was performed automatically on the MR units. Tumor characteristics including maximum dimension $(\mathrm{cm})$, T2-weighted signal appearance (heterogeneity and presence of cystic areas), presence of precontrast T1 shortening or susceptibility in the tumor, T1weighted postcontrast appearance of the noncystic areas, and tumor location were recorded for each tumor by one neuroradiologist (S.F.K.).

\section{Manual Analysis of the Minimum ADC Tumor Value}

A retrospective review and analysis of the brain MR imaging DWI/ADC was independently performed by 2 board-certified neuroradiologists (C.Y.H., S.F.K.) who were blinded to the final pathology. At least 3 regions of interest with size ranging from $1-10 \mathrm{~mm}^{2}$ were placed in the mass at the PACS workstation (Fuji Synapse 3.2.1; Fujifilm, Tokyo, Japan) by use of a manual/freeform region-of-interest tool, and the value was automatically calculated and expressed in $10^{-3} \mathrm{~mm}^{2} / \mathrm{s}$. Sites of region of interest placement were chosen by visual inspection and targeted the darkest signal intensity regions on the ADC map, with the goal of including the lowest ADC portions of the tumor. The entire set of pulse sequences was available during ADC value placement to avoid placing a region of interest on an area of presumed blood products on the basis of intrinsic T1-weighted shortening, T2weighted shortening, or susceptibility effects in the $b=0$ images. Mean ADC values were recorded for each region of interest. The mean ADC values of the 6 ROIs for both neuroradiologists were then averaged and used as the final average minimum value for the tumor. Additionally, mean ADC values from the normal contralateral thalamus and centrum semiovale were obtained in each patient by one neuroradiologist (S.F.K.) to use as an internal reference for comparison. The average minimum ADC value for each tumor and the ratio of the average minimum ADC to the ADC value of the contralateral thalamus $\left(\mathrm{ADC}_{\text {tumor }} / \mathrm{ADC}_{\text {thalamus }}\right)$ or contralateral centrum semiovale $\left(\mathrm{ADC}_{\text {tumor }} / \mathrm{ADC}_{\mathrm{wm}}\right)$ were compared with its respective final tumor pathology WHO grade by use of Spearman $\rho$ correlation. The unpaired 2-tailed Student $t$ test was used to compare for significant differences between the average minimum ADC value and the ADC ratios of the high-grade (WHO grade III and IV) and low-grade tumors (WHO grade I and II). A receiver operating characteristic curve was used to analyze threshold calculations.

\section{Semi-Automated Tumor ADC Value Analysis}

Semi-automated ADC value calculation was performed by use of an in-house script for Matlab (R2011b; MathWorks, Natick, Massachusetts) after a freehand region of interest outlining the entire tumor selected on each axial ADC image for each of the included subjects was performed by a trained medical student (T.A.S.). The ADC images were compared with additional MR imaging from the same study (precontrast and postcontrast T1, T2 sequences) to determine the extent of tumor for selection. Cystic regions of tumor were included, whereas areas of hemorrhage were excluded from the regions of interest. The results were then validated by a board-certified neuroradiologist (A.P.K.). All DICOM ADC voxel values within each of these regions of interest were collected for each individual patient and were then exported to commaseparated value files for import to Excel 2010 (Microsoft, Redwood City, California), with 1 value for each voxel within the tumor, expressed in units $10^{-3} \mathrm{~mm}^{2} / \mathrm{s}$. Individual histograms were generated for the ADC data from each tumor. ADC bin widths of $0.05\left(\times 10^{-3} \mathrm{~mm}^{2} / \mathrm{s}\right)$ were chosen on the basis of the trade-off between detail and noise within the histograms (Fig 1). Normalized summation histograms were generated, 1 for each of the low-grade tumors (WHO grade I and II) and for each of the high-grade tumors (WHO grade III and IV). To create the summation histograms, the ADC histogram data for each individual tumor was normalized by dividing the bin frequencies by the total number of voxels within the tumor, so that each tumor contributed to the tumor group histogram equally, regardless of tumor size. The data in the summation histograms were then normalized by dividing the bin frequencies by the number of tumors in each group so that the group histograms were comparable, eliminating differences that were based on number of tumors in the group. Statistical metrics calculated for the raw ADC data for each tumor included mean, standard deviation, skew, kurtosis, peak height, peak location, and multiple percentiles (5th, 10th, 25th, 50th, 75th, 90th, and 95th). Means and standard deviations were calculated for each statistical metric, grouped by WHO classification. Additionally, scatterplots were generated for each statistical metric grouped by WHO classification. Probability values were calculated for each statistical metric by use of the unpaired, 2-tailed Student $t$ test to evaluate for statistically significant differences between low-grade (WHO I and II) and high-grade (WHO III and IV) results. Also, Spearman $\rho$ correlation was performed between the semi-automated histogram data and the manually obtained average minimum ADC values.

\section{RESULTS}

\section{Supratentorial Tumor Characteristics}

Twenty-two patients were identified; however, 3 patients were excluded. Excluded patients included 1 patient with a complex vascular malformation, 1 patient with an immature teratoma for which no WHO grading was possible, and 1 patient who was excluded because of lack of DWI sequence and ADC map. There 

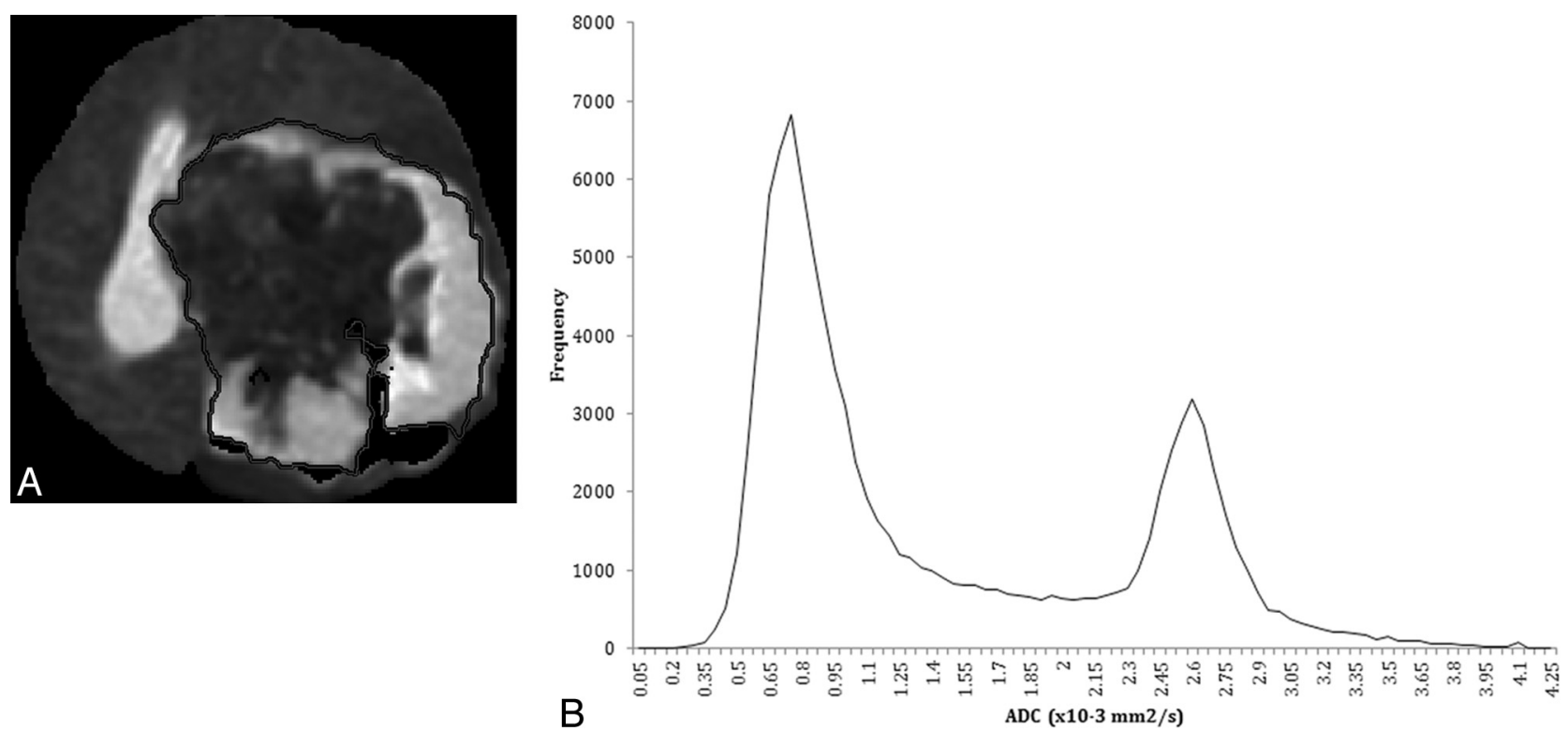

FIG 1. A 4-month-old girl with poorly differentiated carcinoma of the left cerebral hemisphere. A, Representative axial image from ADC map demonstrates manual tracing with general exclusion of large areas of blood products represented by susceptibility artifacts. $B$, Histogram of all included ADC values of the tumor from the semi-automated method.

Table 1: Tumor pathology with corresponding WHO grade and ADC values from manual ROI measurement

\begin{tabular}{|c|c|c|c|c|}
\hline Tumor Type & $\begin{array}{l}\text { WHO } \\
\text { Grade }\end{array}$ & $\begin{array}{l}\text { Average Minimum } \\
\text { ADC } \times 10^{-3} \mathrm{~mm}^{2} / \mathrm{s}\end{array}$ & $\begin{array}{l}\text { ADC Ratio } \\
\text { Thalamus }\end{array}$ & $\begin{array}{c}\text { ADC Ratio } \\
\text { White Matter }\end{array}$ \\
\hline Desmoplastic infantile ganglioglioma & I & 1.63 & 1.68 & 1.50 \\
\hline Desmoplastic infantile ganglioglioma & I & 0.88 & 1.05 & 0.79 \\
\hline Desmoplastic infantile ganglioglioma & I & 0.97 & 1.08 & 0.80 \\
\hline Desmoplastic infantile ganglioglioma & I & 1.14 & 0.87 & 0.76 \\
\hline Choroid plexus papilloma & I & 1.01 & 1.05 & 1.10 \\
\hline Choroid plexus papilloma & I & 0.92 & 1.16 & 0.79 \\
\hline Choroid plexus papilloma & I & 1.31 & 1.53 & 1.23 \\
\hline Pilocytic astrocytoma & I & 0.78 & 0.96 & 0.75 \\
\hline Astrocytoma, focal & $\|$ & 1.71 & 1.50 & 1.35 \\
\hline Pilomyxoid astrocytoma & II & 1.03 & 1.06 & 0.71 \\
\hline Astrocytoma, diffuse & ॥ & 1.12 & 1.27 & 0.98 \\
\hline Anaplastic ependymoma & III & 0.69 & 0.82 & 0.59 \\
\hline Anaplastic ependymoma with tanycytic features & III & 0.54 & 0.57 & 0.47 \\
\hline Choroid plexus carcinoma & III & 1.27 & 0.93 & 0.90 \\
\hline Atypical teratoid/rhabdoid tumor & IV & 0.67 & 0.53 & 0.41 \\
\hline Atypical teratoid/rhabdoid tumor & IV & 0.40 & 0.55 & 0.42 \\
\hline Poorly differentiated carcinoma & IV & 0.56 & 0.45 & 0.32 \\
\hline Poorly differentiated carcinoma & IV & 0.40 & 0.51 & 0.35 \\
\hline Glioblastoma & IV & 0.58 & 0.62 & 0.41 \\
\hline$P$ value, $t$ test between low- and high-grade groups & & .0018 & $<.0001$ & .00042 \\
\hline
\end{tabular}

Note:-All 3 parameters were significant between the difference of the means of the low-grade and high-grade groups. Of note, the choroid plexus carcinoma in our study is an outlier in the high-grade group.

were 9 boys and 10 girls, with a mean age of 4.8 months (range, 1-12 months). Tumor pathologies are listed in Table 1.

T2-weighted images demonstrated a partially cystic mass in 10 cases, whereas the remainder had no significant cystic spaces. Contrast-enhanced T1-weighted images demonstrated 1 nonenhancing tumor (a desmoplastic infantile ganglioglioma), whereas the remainder demonstrated either homogeneous enhancement ( $n=11)$ or heterogeneous enhancement $(n=7)$ of the noncystic areas. Tumor size ranged from $1.3-9.4 \mathrm{~cm}$ (mean, $5.7 \mathrm{~cm}$ ). Interestingly, areas of susceptibility artifact on $b=0$ images and T1 shortening suspected to represent blood products were seen in 6 of the 8 high-grade tumors but in none of the low-grade tumors, though 1 WHO grade I choroid plexus papilloma presented with intraventricular hemorrhage but not intratumoral hemorrhage. Most of the tumors $(n=15)$ had an asymmetric supratentorial location, whereas only 4 tumors were essentially midline tumors ( 3 astrocytomas, 1 choroid plexus papilloma) (Figs 2 and 3).

\section{Manual Analysis of the Average Minimum ADC Tumor Value Versus Tumor Grade}

A significant negative correlation was found between the average minimum $\mathrm{ADC}$ value and the WHO grade (Spearman $\rho=$ $-0.639, P=.0016$ ) (Fig 4 ). There was a significant difference 

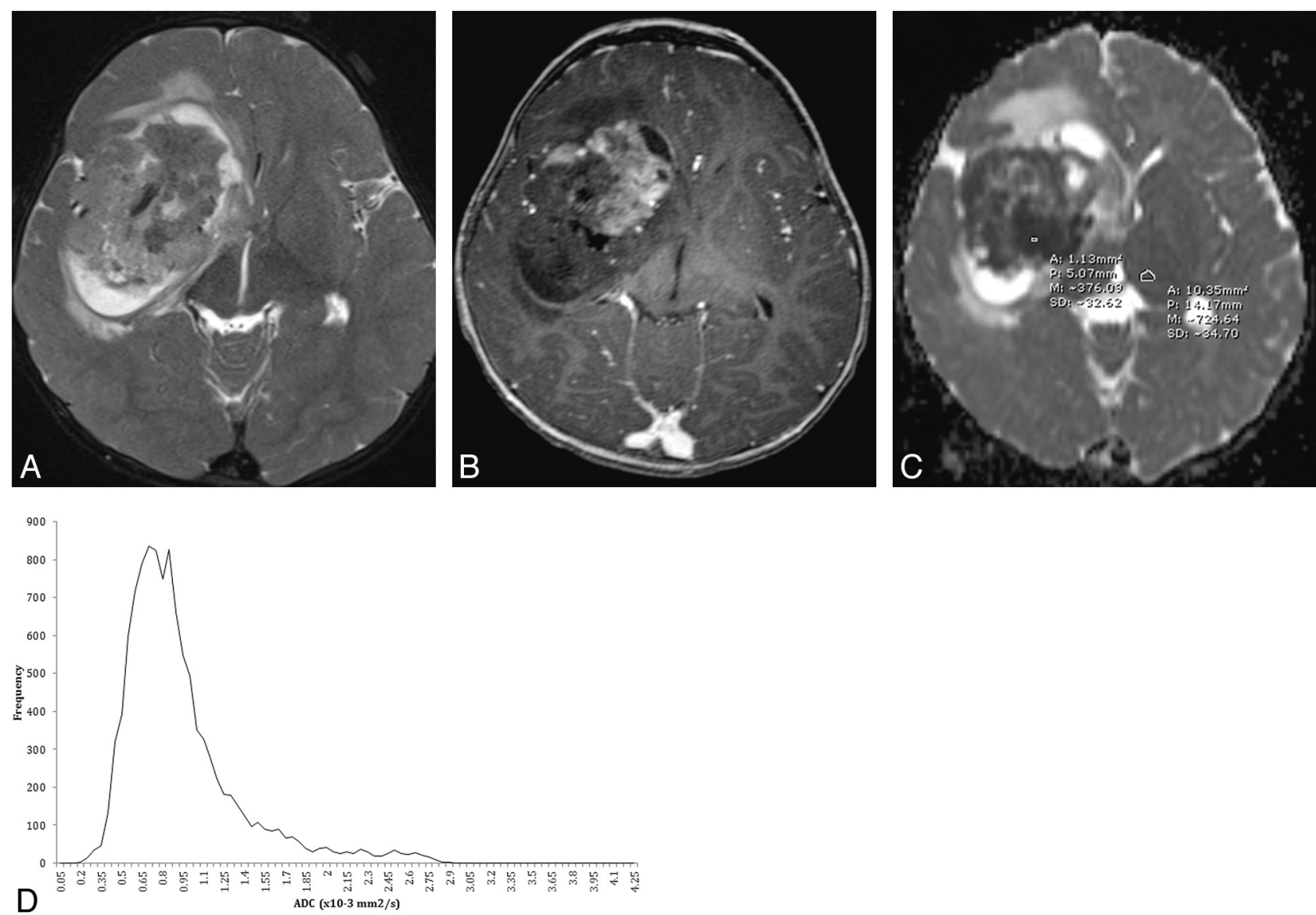

FIG 2. An 11-month-old boy with atypical teratoid/rhabdoid tumor. A, Axial T2-weighted image demonstrates a heterogeneous mass in the right frontal temporal lobe with peripheral cystic change, little peritumoral white matter T2 prolongation, and $B$, heterogeneous enhancement on postcontrast axial 3D T1-weighted image. C, Representative ADC manual region of interest measurement with a small region of interest within the lowest signal portion of the tumor and larger region of interest measuring the contralateral normal thalamus. $D$, Semi-automated histogram for the ADC values of the entire tumor.

between the average minimum $\mathrm{ADC}$ values of the low-grade group $\left(1.14 \times 10^{-3} \mathrm{~mm}^{2} / \mathrm{s} \pm 0.30\right)$ and high-grade group $(0.64 \times$ $\left.10^{-3} \mathrm{~mm}^{2} / \mathrm{s} \pm 0.28\right)(P=.0018)$. Receiver operating characteristic analysis for threshold values (Fig 5) resulted in a 0.9 area under the curve, with $0.687-0.99195 \% \mathrm{CI}$ and significance of $<.0001$. At the furthest distance from the $50 \%$ diagonal, represented by the Youden index (0.875), the proposed threshold between lowgrade versus high-grade tumors in this patient population is $\leq 0.698 \times 10^{-3} \mathrm{~mm}^{2} / \mathrm{s}$ ADC (87.5\% sensitivity: $57.3-99.7,95 \%$ CI, $99.1 \%$ specificity: $71.5-100,95 \% \mathrm{CI})$.

\section{Manual Analysis of ADC Ratios Versus Tumor Grade}

A significant negative correlation was found between $\mathrm{ADC}_{\text {tumor }} /$ $\mathrm{ADC}_{\text {thalamus }}$ ratio and the WHO grade (Spearman $\rho=-0.76, P=$ $.0001)$. There was a significant difference of the $\mathrm{ADC}_{\text {tumor }} /$ $\mathrm{ADC}_{\text {thalamus }}$ mean values in the low-grade group $(1.201 \pm 0.26)$ and high-grade group $(0.623 \pm 0.17)(P=.000034)$.

A significant negative correlation was also found between individual $\mathrm{ADC}_{\text {tumor }} / \mathrm{ADC}_{\mathrm{wm}}$ ratio and the individual WHO grade (Spearman $\rho=-0.74, P=.00025)$. There was a significant difference of the $\mathrm{ADC}_{\text {tumor }} / \mathrm{ADC}_{\mathrm{wm}}$ mean values between the lowgrade group $(0.978 \pm 0.28)$ and high-grade group $(0.484 \pm 0.19)$ $(P=.00042)($ Table 1$)$.

\section{Semi-Automated ADC Analysis}

A significant negative correlation was found between the lowest 5 th percentile ADC value and the WHO grade (Spearman $\rho=$ $-0.663, P=.0002)$ as well as between the lowest 10th percentile ADC value and WHO grade (Spearman $\rho=-0.666, P=.0009$ ). There was a significant difference between the lowest 5 th percentile ADC values in the low-grade group $\left(1.13 \times 10^{-3} \mathrm{~mm}^{2} / \mathrm{s} \pm\right.$ $0.15)$ and the high-grade group $\left(0.78 \times 10^{-3} \mathrm{~mm}^{2} / \mathrm{s} \pm 0.27\right)(P=$ .0020). Similarly, there was a significant difference between the lowest 10th percentile ADC values in the low-grade group $(1.18 \times$ $\left.10^{-3} \mathrm{~mm}^{2} / \mathrm{s} \pm 0.15\right)$ and the high-grade group $\left(0.86 \times 10^{-3}\right.$ $\left.\mathrm{mm}^{2} / \mathrm{s} \pm 0.26\right)(P=.0028)$. The 95 th to 50 th percentile metric, a measure of spread in the upper half of ADC values, had the highest significance $(P=.004)$ between the low $\left(0.82 \times 10^{-3} \mathrm{~mm}^{2} / \mathrm{s} \pm\right.$ $0.43)$ and high-grade $\left(1.43 \times 10^{-3} \mathrm{~mm}^{2} / \mathrm{s} \pm 0.36\right)$ tumors. Histogram representations of $\mathrm{ADC}$ values for low- and high-grade tumors are demonstrated in Fig 6. Conversely, no significant negative correlation was found between the average of all the individual tumor ADC histogram values and the WHO grade (Spearman $\rho=-0.349 ; P=.142$ ) nor a statistical difference of the average ADC histogram values between the low-grade group $\left(1.72 \times 10^{-3}\right.$ $\left.\mathrm{mm}^{2} / \mathrm{s} \pm 0.38\right)$ and the high-grade group $\left(1.46 \times 10^{-3} \mathrm{~mm}^{2} / \mathrm{s} \pm\right.$ $0.34)(P=.138)$. Other metrics such as standard deviation, skew, 

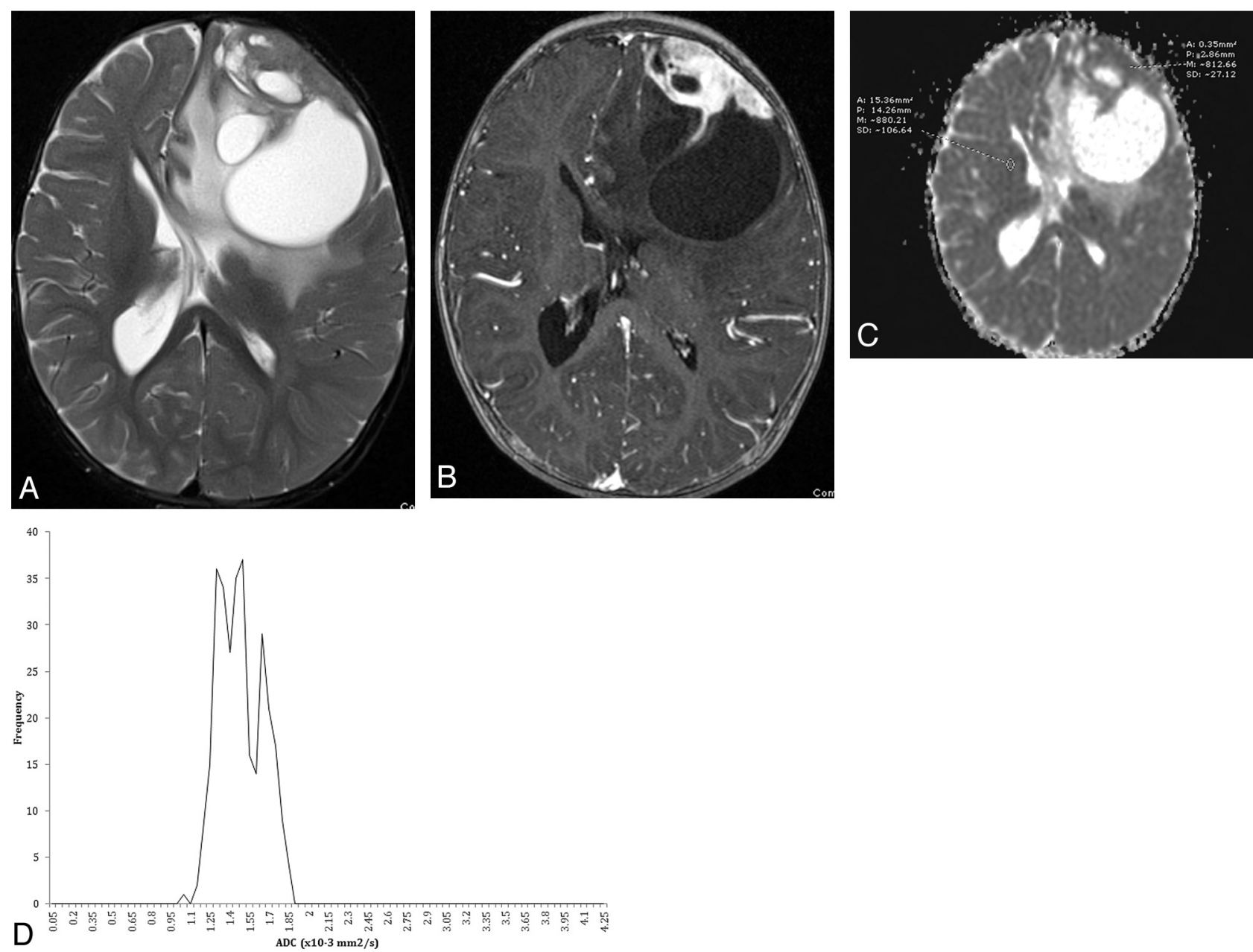

FIG 3. A 12-month-old child with desmoplastic infantile ganglioglioma. A, Axial T2-weighted image demonstrates a heterogeneous mass in the left frontal lobe with cystic change, adjacent T2 prolongation, midline shift, and contralateral ventricular entrapment. $B$, Axial 3D T1-weighted image demonstrates a peripheral enhancing solid component along the dural margin. C, ADC map with representative manual region of interest evaluation within the solid components of the tumor and contralateral white matter. $D$, Semi-automated histogram for the ADC values of the entire tumor.

kurtosis, and peak height were not significant, whereas peak location and 25th and 50th percentile bins demonstrated mild significance (Table 2).

When comparing the average minimum ADC value obtained from the manual method versus the lowest 5 th and 10th percentile ADC values from the semi-automated method, there was a statistically significant correlation between the values (Spearman $\rho=0.95 ; P<.000001)$ and (Spearman $\rho=0.92, P<.000001)$, respectively.

\section{DISCUSSION}

In our selection of patients, we chose to limit the age of the patients with supratentorial tumors to 1 year of life on the basis of the higher incidence of supratentorial tumors in this age group and to encompass congenital tumors. ${ }^{1,4}$ In our patient population, a diagnosis on the basis of location could be highly suspected for some pathologies, such as a clearly intraventricularly located choroid plexus tumor or an optic pathway astrocytoma; however, many of the tumors that we encountered demonstrated a challenging diagnosis with a common imaging appearance of a large, cystic, and solid enhancing mass that is asymmetric to one hemisphere. Additionally, the large size of the mass may result in dif- ficulty determining intraventricular origin. In a series of neonatal brain tumors, Buetow et $\mathrm{al}^{4}$ described lesions that occupied more than one-third of the intracranial volume in $75 \%$ of the cases. Comi et $\mathrm{al}^{5}$ analyzed neuroradiologic findings in 40 children younger than 3 years of age with intracranial ependymomas: mean tumor diameter at diagnosis was approximately $4.2 \mathrm{~cm}$. Similarly, we encountered a mean tumor diameter of $5.7 \mathrm{~cm}$. When faced with a large, solid, and cystic enhancing supratentorial mass in a pediatric patient in the first year of life, assessment of the tumor ADC characteristics provides additional information in determining tumor grade.

T1- and T2-weighted without and with contrast MR imaging sequences (hereafter termed "conventional MR imaging") have been shown to be insufficient for differentiation and grading of brain tumors in part because peritumoral edema, enhancement, necrosis, or mass effect may be seen with both high-grade or lowgrade tumors, and the enhancing portions of tumors may not always reflect the most malignant part of the tumor. ${ }^{6,7}$ Tumoral enhancement may be caused by disruption of the blood-brain barrier or from tumoral vascular proliferation. These 2 are independent of each other, and consequently the enhancement pat- 


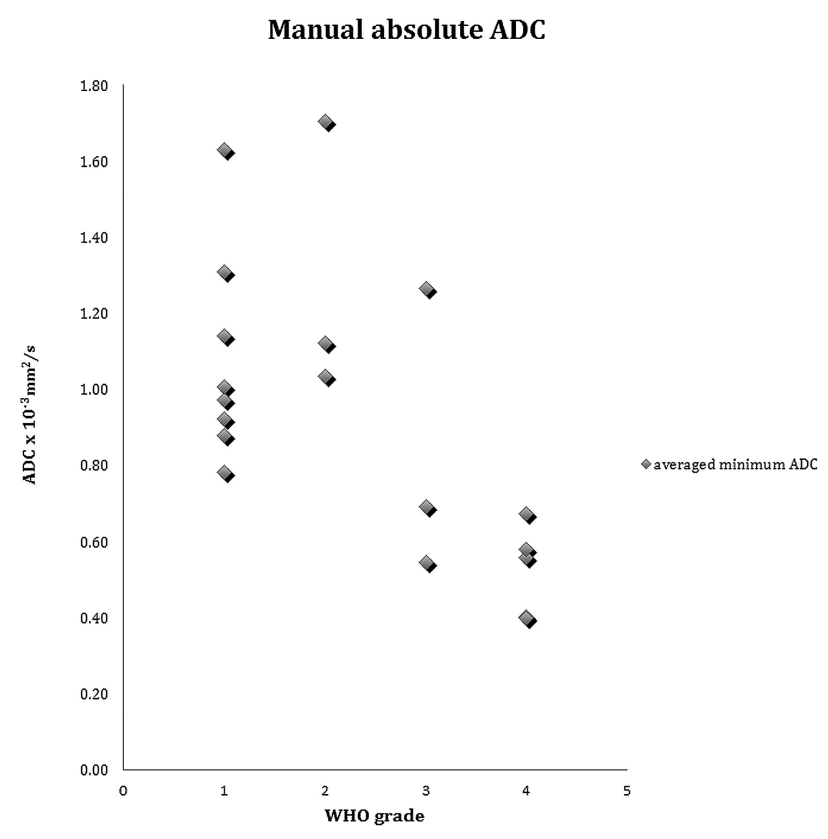

FIG 4. Scatterplot of the average absolute minimum ADC for all tumors by $\mathrm{WHO}$ grading.

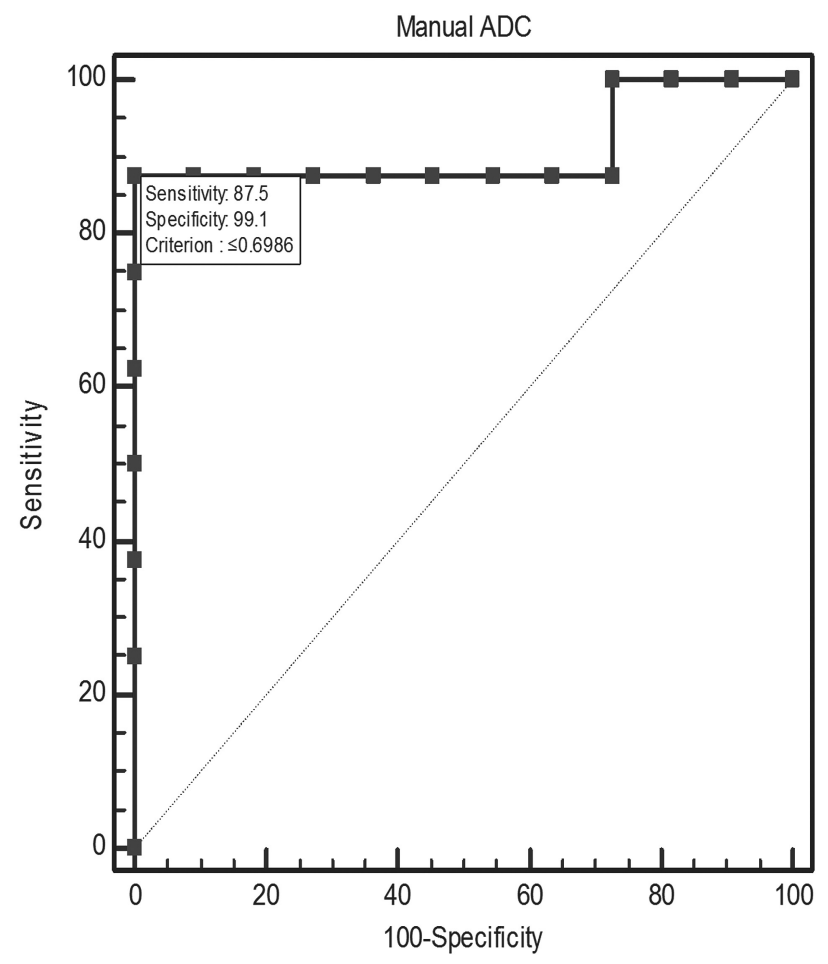

FIG 5. Receiver operating characteristic curve for manual absolute ADC demonstrates a significant area above the $50 \%$ diagonal. Threshold according to the Youden index is $\leq 0.698 \times 10^{-} 3 \mathrm{~mm}^{2} / \mathrm{s}$ for WHO grade III and IV tumors versus grade I and II tumors.

tern of a tumor is not always reliable for differentiating high- and low-grade tumors. ${ }^{6,7}$ Consequently, diffusion and perfusion techniques that improve specificity of tumor diagnosis and grading remain valuable.

Diffusion imaging has been shown to demonstrate particular utility in neuroimaging for a wide range of processes. Diffusion imaging can detect areas of acute ischemia that leads to limitation in molecular movement caused by cytotoxic edema. A similar application of diffusion imaging has been applied for evaluation of brain tumors that may alter the molecular motion of water from structural alterations caused by the destruction of neuronal architecture, tumor cellularity causing reduction of the interstitial space, and vasogenic edema, which may increase the extracellular space. Although studies of adult and pediatric tumors have demonstrated that increasing tumor cellularity often leads to increasing signal intensity on DWI and hypointensity on ADC maps, ${ }^{8-11}$ one study evaluating oligodendrogliomas did not demonstrate such a relationship. ${ }^{12}$ Multiple factors in addition to cellular density probably contribute to differences in ADC values; nonetheless, these differences in $\mathrm{ADC}$ values can provide information to differentiate pediatric tumors.

Diffusion imaging with ADC maps has demonstrated utility in pediatric patients with cerebellar tumors. ${ }^{3}$ In the analysis by Rumboldt et al, ${ }^{3}$ ADC maps could reliably differentiate the common pediatric cerebellar tumors of ependymoma, medulloblastoma, and pilocytic astrocytoma. However, subsequent studies demonstrate overlap of ADC values of these posterior fossa tumors, particularly ependymomas, indicating that diffusion imaging is a valuable but imperfect tool for cerebellar tumor differentiation. ${ }^{13,14}$ The pathology of the supratentorial tumors commonly encountered in the first year of life includes a wide spectrum of low- and high-grade tumor types unlike cerebellar tumors. Furthermore, there is potential for tumors for which it may be difficult to determine a specific pathologic diagnosis, as was the case in 2 of our WHO grade IV tumors. Whereas highgrade diagnoses such as primitive neuroectodermal tumor, atypical teratoid/rhabdoid tumor, and choroid plexus carcinoma were considered, the final pathologic diagnosis was "poorly differentiated carcinoma, WHO grade IV." The combination of a wide range of pathologies and potential difficulty in determining a specific pathologic diagnosis illustrates the potential difficulty with specific tumor diagnosis. Pediatric cerebellar tumors may have additional supportive conventional MR imaging appearance such as the classically described cyst and solid enhancing nodule pattern of a pilocytic astrocytoma or extension through the foramen Luschka of an ependymoma. Although desmoplastic infantile gangliogliomas have been typically described with a peripheral, dural-based enhancing nodule and cyst generally, supratentorial tumors in the first year of life can present with large, heterogeneous, enhancing masses that have very similar imaging findings, whether low- or high-grade.

Recognizing these potential obstacles, our goal was to determine if ADC values demonstrate utility for differentiating lowgrade and high-grade supratentorial tumors in pediatric patients presenting in the first year of life. To our knowledge, this is the first study assessing ADC values of supratentorial brain tumors in pediatric patients presenting in the first year of life. In this study, we were able to demonstrate that careful analysis of the ADC maps of supratentorial brain tumors in children younger than 1 year of age results in statistically significant differentiation of high- and low-grade tumors. We chose to use the lowest ADC value in the manual region of interest placement because of the importance of tumor differentiation, which primarily involves identifying areas of highest cellularity that result in lower ADC 

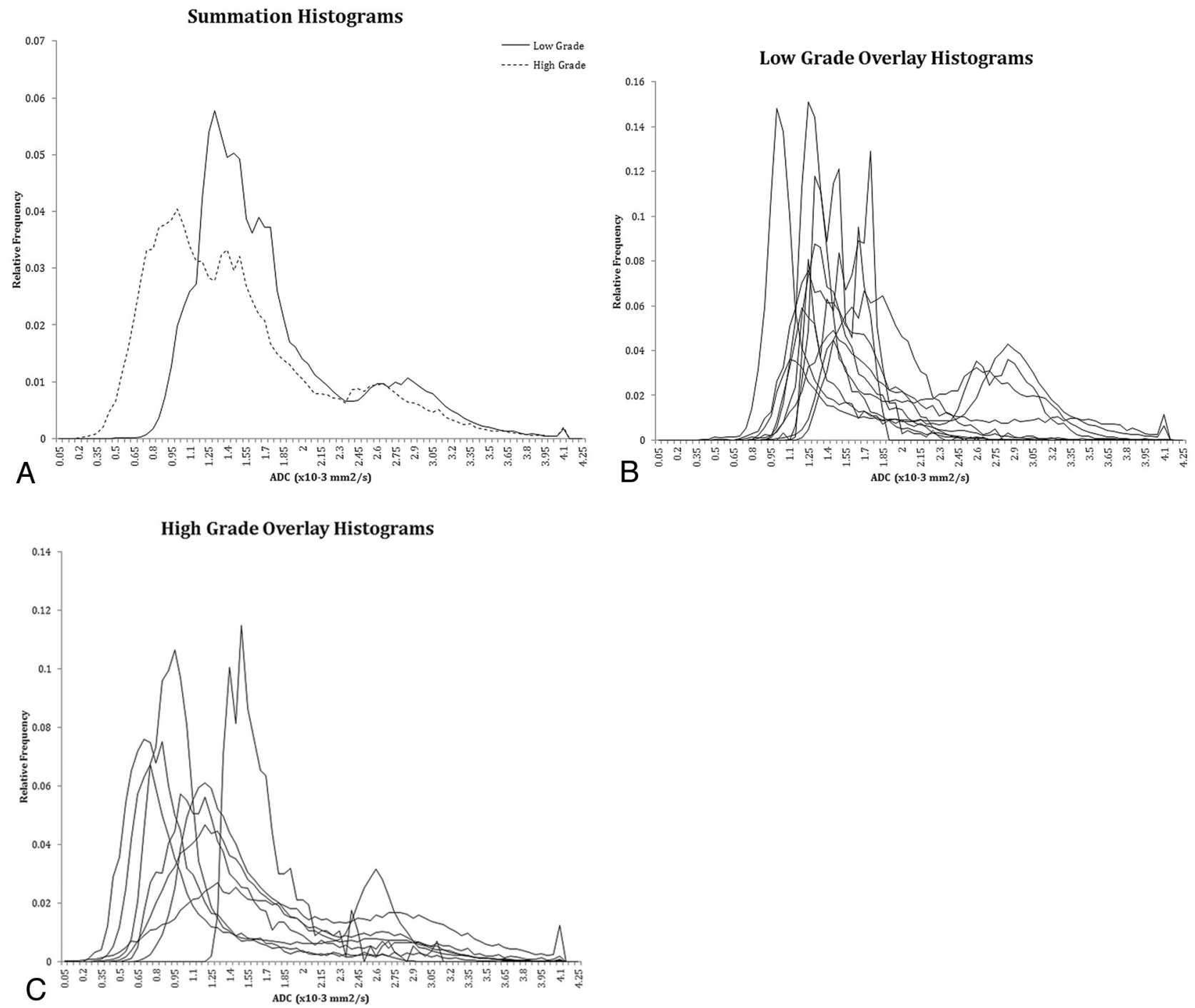

FIG 6. $A$, Summation semi-automated histograms of ADC values of all low-grade tumors compared with high-grade tumors. $B$, Overlay histograms of all low-grade tumors. C, Overlay histograms of all high-grade tumors.

values. We developed a semi-automated method that uses a computer algorithm to collect ADC values within a defined tumor region as a potentially more unbiased method and a method to validate the manual region of interest placement. The semi-automated method demonstrates statistically significant differences in individual tumor grades for the lowest 5 th and 10th percentile ADC values obtained from each individual tumor versus a lack of a statistically significant difference between the average tumor $\mathrm{ADC}$ value and the tumor grade. This, in addition to the lack of significance in other parameters such as standard deviation, skew, and kurtosis, mathematically corroborates the heterogeneous nature of these tumors and the wide range of $\mathrm{ADC}$ values possible. Although choosing to include cystic areas does skew the average ADC value higher, including the whole of the tumor decreases selection bias while still incorporating the lowest ADC values as a metric. Furthermore, some tumors with heterogeneous cysts may be difficult to easily partition from the solid portions of the tumor. This inclusion of cystic structures probably led to significance in the 95th to 50th percentile metric, indicating that low-grade tumors tend to be more homogeneous, with less spread in the upper ranges of ADC values, whereas high- grade tumors are more heterogeneous in this ADC range. Finally, the manual method of identifying the lowest $\mathrm{ADC}$ value in the tumor was validated as a method when compared with the semiautomated lowest 5 th and 10 th percentile ADC values. This indicates that the more simple manual approach, applicable to all radiologists with reading stations allowing region of interest measurements, is not only sufficient but demonstrates more significance because of lower $P$ values of the manual metrics compared with the semi-automated metrics for grading analysis of these tumors.

We analyzed both the average minimum ADC value as well as a ratio of the average minimum tumor $\mathrm{ADC}$ value to the $\mathrm{ADC}$ value of the contralateral normal thalamus and to the contralateral normal centrum semiovale. Because the ADC values of normally developing brain decrease with increasing age, especially in the white matter with normal myelin maturation, use of the average minimum ADC value may be preferred over ADC ratios despite the statistical significance obtained for the ADC ratios in this study. ${ }^{15-17}$ However, ADC ratios were performed because our data were acquired from different MR units, and a potential in- 
Table 2: Semi-automated metrics including mean and standard deviations for low- and high-grade tumors

\begin{tabular}{|c|c|c|c|c|c|}
\hline & \multicolumn{2}{|c|}{ Low-Grade } & \multicolumn{2}{|c|}{ High-Grade } & \multirow[b]{2}{*}{$\begin{array}{l}P \text { Value } \\
\text { ( } t \text { test) }\end{array}$} \\
\hline & $\begin{array}{c}\text { Mean } \\
\left(\times 10^{-3} \mathrm{~mm}^{2} / \mathrm{s}\right)\end{array}$ & $\begin{array}{c}\text { Standard } \\
\text { Deviation } \\
\left(\times 10^{-3} \mathrm{~mm}^{2} / \mathrm{s}\right)\end{array}$ & $\begin{array}{c}\text { Mean } \\
\left(\times 10^{-3} \mathrm{~mm}^{2} / \mathrm{s}\right)\end{array}$ & $\begin{array}{c}\text { Standard } \\
\text { Deviation } \\
\left(\times 10^{-3} \mathrm{~mm}^{2} / \mathrm{s}\right)\end{array}$ & \\
\hline Average & 1.717 & 0.377 & 1.459 & 0.341 & .138 \\
\hline Standard deviation & 0.458 & 0.247 & 0.616 & 0.189 & .135 \\
\hline Skew & 0.803 & 0.753 & 1.372 & 0.704 & .111 \\
\hline Kurtosis & 1.129 & 2.515 & 1.980 & 2.754 & .501 \\
\hline Peak location & 1.518 & 0.493 & 1.075 & 0.275 & $.023^{\mathrm{a}}$ \\
\hline Peak height, normalized & 0.088 & 0.041 & 0.069 & 0.029 & .258 \\
\hline 5th Percentile & 1.13 & 0.151 & 0.778 & 0.267 & $.007^{\mathrm{a}}$ \\
\hline 10th Percentile & 1.18 & 0.151 & 0.858 & 0.260 & $.008^{\mathrm{a}}$ \\
\hline 25th Percentile & 1.32 & 0.180 & 1.016 & 0.278 & $.018^{\mathrm{a}}$ \\
\hline 50th Percentile & 1.68 & 0.453 & 1.270 & 0.349 & $.037^{\mathrm{a}}$ \\
\hline 75th Percentile & 2.01 & 0.598 & 1.792 & 0.564 & .411 \\
\hline 90th Percentile & 2.32 & 0.681 & 2.363 & 0.591 & .903 \\
\hline 95th Percentile & 2.50 & 0.705 & 2.696 & 0.541 & .518 \\
\hline 95th to 5th Percentile & 1.377 & 0.704 & 1.918 & 0.560 & .080 \\
\hline 50th to 5th Percentile & 0.555 & 0.425 & 0.491 & 0.273 & .695 \\
\hline 95th to 50th Percentile & 0.821 & 0.434 & 1.426 & 0.362 & $.004^{\mathrm{a}}$ \\
\hline
\end{tabular}

a Significant $P$ value.

tervendor variance in absolute ADC values has been previously reported. ${ }^{18}$

Limitations of this study include the potential for selection bias of the ADC region of interest placement by observation of the other conventional MR images and the total number of patients. We chose not to blind the placement of the region of interest to the remainder of the anatomic sequences to prevent placing a region of interest on suspected blood products as well as to simulate region of interest placement that may occur in clinical practice. The semi-automated method validates the manual placement of the ADC, though this also has the potential for selection bias on the basis of the decision of placement of the borders of the tumor. Our study is the largest, to our knowledge, within the current literature to assess diffusion imaging for intracranial tumors within the first year of life. However, because these tumors are rare, the number of total patients in this study limits our ability to determine whether a specific pathology could be determined with ADC values within the low- or high-grade groups, as well as separating low- and high-grade tumors of similar pathology. An example of this is the 4 choroid plexus tumors in our study. The average ADC value of the 1 choroid plexus carcinoma was within the range of the 3 choroid plexus papillomas in our study group. Although this may be an outlier, only a single small study within the literature that used MR spectroscopy suggested a potential differentiation method of elevated myo-inositol levels in choroid plexus papillomas versus carcinomas. ${ }^{19}$ In the differentiation of specific tumor pathology within the high-grade or low-grade groups, we suspect that even with a larger number of patients, differentiation of tumor pathology may not be possible with ADC values, similar to the inability of diffusion imaging to differentiate medulloblastoma from atypical teratoid/rhabdoid tumor (both WHO grade IV tumors). ${ }^{20}$ Similarly, WHO grading may not always predict biologic behavior and ultimately outcome $\mathrm{e}^{21,22}$; however, this does not detract from the utility of ADC values in the characterization and diagnosis of these supratentorial tumors and provides a foundation for the radiologist to establish a differential diagnosis.

\section{CONCLUSIONS}

Diffusion imaging with ADC maps provides valuable diagnostic information when brain tumors are evaluated. Manually obtaining the minimum ADC value within the tumor with region of interest measurements can be simple and reliable. Despite the considerable heterogeneity of supratentorial tumors presenting in the first year of life, there is a trend between decreasing tumoral ADC value with higher WHO grade, and the lowest tumoral ADC values can reliably differentiate low- and high-grade tumors, leading to improved diagnosis and facilitating preoperative planning.

\section{REFERENCES}

1. Sala F, Colarusso E, Mazza C, et al. Brain tumors in children under 3 years of age: recent experience (1987-1997) in 39 patients. Pediatr Neurosurg 1999;31:16-26

2. Sugahara T, Korogi Y, Kochi M, et al. Usefulness of diffusionweighted MRI with echo-planar technique in the evaluation of cellularity of gliomas. J Magn Reson Imaging 1999;9:53-60

3. Rumboldt Z, Camacho DL, Lake D, et al. Apparent diffusion coefficients for differentiation of cerebellar tumors in children. AJNR Am J Neuroradiol 2006;27:1362-69

4. Buetow PC, Smirniotopoulos JG, Done S. Congenital brain tumors: a review of 45 cases. AJR Am J Roentgenol 1990;155:587-93

5. Comi AM, Backstrom JW, Burger PC, et al. Clinical and neuroradiologic findings in infants with intracranial ependymomas. Pediatr Neurol 1998;18:23-29

6. Knopp EA, Cha S, Johnson G, et al. Glial neoplasms: dynamic contrast enhanced T2* weighted MR imaging. Radiology 1999;211:791-98

7. Law M, Yang S, Wang H, Glioma grading: sensitivity, specificity, and predictive values of perfusion MR imaging and proton MR spectroscopic imaging compared with conventional MR imaging. AJNR Am J Neuroradiol 2003;24:1989-98

8. Gauvain KM, McKinstry RC, Mukherjee P, et al. Evaluating pediatric brain tumor cellularity with diffusion-tensor imaging. AJR Am J Roentgenol 2001;177:449-54

9. Gupta RK, Cloughesy TF, Sinha U, et al. Relationships between choline magnetic resonance spectroscopy, apparent diffusion coefficient and quantitative histopathology in human glioma. J Neurooncol 2000;50:215-26

10. Guo AC, Cummings TJ, Dash RC, et al. Lymphomas and high-grade 
astrocytomas: comparison of water diffusibility and histologic characteristics. Radiology 2002;224:177-83

11. Yamashita $Y$, Kumabe T, Higano S, et al. Minimum apparent diffusion coefficient is significantly correlated with cellularity in medulloblastoma. Neurol Res 2009;31:940-46

12. Jenkinson MD, du Plessis DG, Smith TS, et al. Cellularity and apparent diffusion coefficient in oligodendroglial tumours characterized by genotype. J Neurooncol 2010;96:385-92

13. Gimi B, Cederberg K, Derinkuyu B, et al. Utility of apparent diffusion coefficient ratios in distinguishing common pediatric cerebellar tumors. Acad Radiol 2012;19:794-800

14. Jaremko JL, Jans LB, Coleman LT, et al. Value and limitations of diffusion-weighted imaging in grading and diagnosis of pediatric posterior fossa tumors. AJNR Am J Neuroradiol. 2010;31:1613-16

15. Morriss MC, Zimmerman RA, Bilaniuk LT, et al. Changes in brain water diffusion during childhood. Neuroradiology 1999;41: 929-34

16. Sener RN. Diffusion MRI apparent diffusion coefficient (ADC) values in the normal brain and a classification of brain disorders based on ADC values. Comput Med Imaging Graph 2001;25:299-326
17. Lovblad KO, Schneider J, Ruoss K, et al. Isotropic apparent diffusion coefficient mapping of postnatal cerebral development. Neuroradiology 2003;45:400-03

18. Sasaki M, Yamada K, Watanabe Y, et al. Variability in absolute apparent diffusion coefficient values across different platforms may be substantial: a multivendor, multi-institutional comparison study. Radiology 2008;249:624-30

19. Krieger MD, Panigrahy A, McComb JG, et al. Differentiation of choroid plexus tumors by advanced magnetic resonance spectroscopy. Neurosurg Focus 2005;18:E4

20. Koral K, Gargan L, Bowers DC, et al. Imaging characteristics of atypical teratoid-rhabdoid tumor in children compared to medulloblastoma. AJR Am J Roentgenol 2008;190:809-14.

21. Gravendeel LA, Kouwenhoven MC, Gevaert O, et al. Intrinsic gene expression profiles of gliomas are a better predictor of survival than histology. Cancer Res 2009;69:9065-72

22. Zamecnik J, Snuderl M, Eckschlager T, et al. Pediatric intracranial ependymomas: prognostic relevance of histological, immunohistochemical, and flow cytometric factors. Mod Pathol 2003; 16:980-91 